\title{
ACTIVIST POLICY AND MACROECONOMIC INSTABILITY *
}

\author{
C.F. BAUM \\ Boston College, Chestnut Hill, MA 02167, USA \\ E.P. HOWREY \\ The University of Michigan, Ann Arbor, MI 48109, USA
}

Received 4 May 1982

This paper investigates the consequences of activist monetary policy in a small, aggregate macroeconomic model which embodies traditional hypotheses of price and output determination. It is shown that deterministic control problems with inflation and full employment as objectives may be solved, but multiple-target stochastic control problems will lead to instability in both the targets and the policy instrument.

\section{Introduction and summary}

The use of conventional macroeconomic models to analyze the effects of monetary and fiscal policies has been the subject of considerable discussion and controversy in recent years. The strong forms of the rational expectations and natural rate hypotheses suggest that there is limited scope for active stabilization policies and perhaps, also, for the use of traditional macroeconometric models. These challenges are typically countered with the argument that the empirical evidence, as interpreted by Gordon (1979), Nelson (1981), Makin (1981), and Mishkin (1982), for example, is not sufficiently compelling to justify abandonment of the orthodox approach to macroeconomic policy analysis.

* This paper is derived from Baum and Howrey (1981). We appreciate the comments of several of the SEDC Conference participants, as well as those of Saul Hymans, Art Rolnick, Jim Anderson and Bruce Smith. The usual caveat applies.

0165-1765/83/0000-0000/\$03.00 (c) 1983 North-Holland 
I The purpose of this note is to point out a potential difficulty with the conduct of activist stabilization policy within the context of a traditional macroeconomic model. In a model with conventional price and output adjustment equations, we find that in the absence of stochastic disturbances there need not be any difficulty in pursuing active stabilization policy to achieve both full employment and price stability. However. when stochastic disturbances are introduced into this congenial environment, optimal control of inflation and unemployment inevitably leads to stochastic instability of both targets and instruments. Thus, even in a traditional macro framework, the tradeoff between inflation and unemployment may not be exploitable on a continuing basis in a world of recurrent price and output shocks.

\section{The model}

The model we analyze is a condensed version of the model in Baum and Howrey (1981). Its basic elements include an aggregate demand curve, an aggregate output equation and a price adjustment equation. These three equations are written in log-linear form as

$$
\begin{aligned}
& Y=\alpha_{0}+\alpha(M-P), \\
& Q=Q_{-1}+\beta\left(Y-Q_{-1}\right), \\
& P=P_{-1}+\gamma\left(Q-Q^{*}\right),
\end{aligned}
$$

where $Y$ is the logarithm of real aggregate demand, $M$ is the logarithm of the stock of money, $P$ is the logarithm of the price level, $Q$ is the logarithm of real output, and $Q^{*}$ is the logarithm of potential output. The aggregate demand equation can be viewed as a standard reduced form equation obtained from the IS-LM framework in which real aggregate demand depends on (among other things) real money balances. The output equation is a partial adjustment equation in the production smoothing tradition in which production is assumed to respond to excess aggregate demand, $\left(Y-Q_{-1}\right)$. Finally, a standard Phillips curve is used to relate the price level to the gap between potential and actual output, similar to that studied by Frye and Gordon (1980).

In order to simplify the development of the basic result, we suppose that $\beta=1$, combine eqs. (1) and (2) via the equilibrium condition $Y=Q$, 
and append stochastic disturbance terms to the equations to obtain

$Q=\alpha_{0}+\alpha(M-P)+U_{Q}$,

$p=\gamma R+U_{p}$

where $p=\Delta P$ is the rate of price inflation and $R=\left(Q-Q^{*}\right)$ is the gap between actual and potential output. These two variables correspond to the targets of price stability and full employment. To analyze their behavior, we derive the fundamental dynamic equations [Kmenta (1971, p. 591)] for $R$ and $p$ :

$$
\begin{aligned}
& (1-\lambda L) R=\alpha \lambda m-\lambda q^{*}+\lambda \Delta U_{Q}-\alpha \lambda U_{P}, \\
& (1-\lambda L) p=\alpha \gamma \lambda m-\gamma \lambda q^{*}+\gamma \lambda \Delta U_{Q}+\lambda \Delta U_{P},
\end{aligned}
$$

where $m=\Delta M, q^{*}=\Delta Q^{*}, \lambda=1 /(1+\alpha \gamma)$, and $L$ is the lag operator.

It is readily apparent from these equations that, in the absence of output and price disturbances, setting $m=\alpha^{-1} q^{*}$ yields an equilibrium with full employment ( $R=0$ ) and a constant price level $(p=0)$ which is dynamically stable, since $0<\lambda<1$ provided that $\alpha>0$ and $\gamma>0$. Deviations from this equilibrium resulting from price and output disturbances will be stable, in the sense of possessing a finite variance, if the disturbance processes $\left\{U_{P}\right\}$ and $\left\{U_{Q}\right\}$ have finite variances. A common assumption, consistent with a broad range of empirical findings, is that $\left\{\Delta U_{P}\right\}$ and $\left\{\Delta U_{Q}\right\}$ are serially independent. In this case, the rate of inflation is stochastically stable, but the gap is stochastically unstable since $\left\{U_{P}\right\}$ follows a random walk. We now suppose that $q^{*}, \alpha$, and $\gamma$ are known, and consider the certainty equivalent control problem for this model.

\section{Activist stabilization policy}

If $m$ is set each period to minimize

$$
C=E\left[K_{1} p^{2}+K_{2} R^{2}\right]
$$

the following system, including the endogenous policy, is obtained:

$$
R=\pi_{11} R_{-1}+\pi_{12} P_{-1}+W_{R},
$$


$p=\pi_{21} K_{-1}+\pi_{22} p_{-1}+W_{P}$,

$m=\pi_{31} R_{-1}+\pi_{32} p_{-1}+\alpha^{-1} q^{*}+W_{m}$.

The parameters and disturbances in this system are defined by

$$
\begin{aligned}
& \pi_{11}=\lambda\left(1-\delta K_{2}\right), \\
& \pi_{12}=-\gamma \delta \lambda K_{1}, \\
& \pi_{21}=-\gamma \delta \lambda K_{2}, \\
& \pi_{22}=\lambda\left(1-\gamma^{2} \delta K_{1}\right), \\
& \pi_{31}=-\alpha^{-1} \delta K_{2}, \\
& \pi_{32}=-\alpha^{-1} \gamma \delta K_{2}, \\
& W_{R}=\lambda V_{Q}-\lambda\left(\alpha-\gamma \delta K_{1} L\right) U_{P}-\lambda \delta\left(\gamma K_{1}-\alpha K_{2}\right) \tilde{U}_{P}, \\
& W_{p}=\gamma \lambda V_{Q}+\lambda\left(1-\left(1-\gamma^{2} \delta K_{1}\right) L\right) U_{P}-\gamma \delta \lambda\left(\gamma K_{1}-\alpha K_{2}\right) \tilde{U}_{P}, \\
& W_{m}=-\alpha^{-1} \tilde{U}_{Q}-\delta\left(\alpha^{-1} \gamma K_{1}-K_{2}\right) \tilde{U}_{p}+\alpha^{-1} L U_{Q}+\alpha^{-1} \gamma \delta K_{1} L U_{P},
\end{aligned}
$$

where $\delta=\left(\gamma^{2} K_{1}+K_{2}\right)^{-1}, \tilde{U}_{p}\left(\tilde{U}_{Q}\right)$ is the expected value of $U_{P}\left(U_{Q}\right)$ at the beginning of the decision period, $V_{Q}$ is the unanticipated part of $U_{Q}$, and $L$ is the lag operator.

It will be noted that $W_{R}, W_{p}$, and $W_{m}$ will contain $U_{P}, \tilde{U}_{P}$ or both whenever both $K_{1}$ and $K_{2}$ are non-zero. It follows that if $U_{P}$ follows a random walk, as empirical research suggests, any attempt to control both inflation and unemployment - irregardless of relative weights in the loss function - leads to stochastic instability of both the targets and the instrument.

Two special cases of interest are obtained by placing only one target in the loss function. With $K_{1}=1, K_{2}=0$, inflation is the only target, and the controlled system takes the form

$$
\begin{aligned}
& m=\gamma \lambda q^{*}-\lambda p_{-1}-\gamma \lambda\left(\tilde{U}_{Q}-L U_{Q}\right)-\lambda\left(\tilde{U}_{P}-L U_{P}\right), \\
& p=\gamma \lambda V_{Q} \text { । } \lambda V_{P},
\end{aligned}
$$


$R=\lambda V_{Q}+\gamma^{-1} \lambda V_{P}-\lambda(1-\lambda L){ }^{1}\left(\gamma^{-1} \Delta U_{P}+\alpha U_{P}\right)$,

where $V_{P}$ is the unanticipated part of $U_{P}$. If $U_{Q}$ and $U_{P}$ follow random walks, then $\tilde{U}_{Q}=L U_{Q} ; \tilde{U}_{P}=L U_{p}$; both $m$ and $p$ are stochastically stable, but $R$ is stochastically unstable, because it depends upon $U_{P}$.

If the monetary instrument is set to maintain full employment, $K_{1}=0$, $K_{2}=1$, and the controlled system becomes

$$
\begin{aligned}
& m=\alpha^{-1} q^{*}+\alpha^{-1}(1-\lambda) V_{Q}-(1-\lambda) V_{P}-\alpha^{-1} \Delta U_{Q}+U_{P}, \\
& p=\gamma \lambda V_{Q}-\alpha \gamma \lambda V_{P}+U_{P}, \\
& R=\lambda V_{Q}-\alpha \lambda V_{P} .
\end{aligned}
$$

In this case, $R$ differs from zero in any period by a serially uncorrelated random disturbance. Both $m$ and $p$ are stochastically unstable if $U_{p}$ follows a random walk.

The results of this analysis may be summarized as follows. With price level disturbances following a random walk, if $p$ is controlled, $R$ will be unstable; if $R$ is controlled, $p$ will be unstable; if both $p$ and $R$ are controlled, both will be unstable. Moreover, control of $R$ alone or control of $p$ and $R$ together leads to stochastic instability of the instrument. In such a world, it would not be surprising if the monetary authority exhibited schizophrenic behavior!

One might conjecture that this pervasive instability is due to the absence of a weight on the instrument, $m$. The objective function (8) may be expanded to include a weight $K_{3}$ on $\left(m-m^{*}\right)^{2}$, the deviation of money from its desired path. Alternatively, a derivative stabilization of $m$ could be implemented with the term $K_{3}\left(m-m_{-1}\right)^{2}$. In either case, it may be shown that the qualitative result remains: any set of non-zero weights $\left[K_{1}, K_{2}, K_{3}\right]$ will produce an unstable system. The use of proportional control of the instrument will introduce monotonic terms in the characteristic equation, whereas the derivative control of $m$ may potentially introduce oscillatory behavior. In the end, all such systems are qualitatively identical to the system described above.

This strong instability result limiting the scope for multitarget activist policy is the result of our assumptions regarding the disturbance process: namely, that $U_{P}$ is a random walk. If this strong assumption is modified to

$(1-\rho L) U_{P}=V_{P}$, 
where $\rho$ is slightly less than unity, the extreme result disappears. The infinite variances will now be finite, but perhaps still unacceptably large. Activist policy may in this case be technically feasible, but may have little to offer the architect of stabilization policy.

\section{Conclusions}

The characteristics of activist policy in the context of a small, traditional macroeconomic model were examined. We found that substantial improvement in the inflation rate or the employment rate could be achieved through monetary control, even in the presence of unstable price level shocks. However, if monetary policy is designed to maintain full employment without inflation, unstable price shocks lead to chaos.

\section{References}

Baum, C.F. and E.P. Howrey, 1981, An examination of postwar U.S. stabilization policy: Monetary and fiscal policy in an accelerationist world, Presented at the Third Economics and Control Conference of the Society for Economic Dynamics and Control (Lyngby).

Frye, J. and R.J. Gordon, 1980, The variance and acceleration of inflation in the 1970's: Alternative explanatory models and methods, Working paper (Northwestern University, Evanston, IL).

Gordon, R.J., 1979, New evidence that fully anticipated monetary changes influence real output after all, Discussion paper no. 369 (Northwestern University, Evanston, IL).

Kmenta, Jan, 1971, Elements of econometrics (Macmillan, New York).

Makin, John, 1981, Anticipated money and real economic activity, NBER Conference on Inflation and Financial Markets (Cambridge, MA).

Mishkin, F.S., 1982, Does anticipated monetary policy matter? An econometric investigation, Journal of Political Economy 90, 22-51.

Nelson, C.R., 1981, Adjustment lags versus information lags: A test of alternative explanations of the Phillips curve phenomenon, Journal of Money. Credit and Banking 13. $1-11$. 